\title{
DOCTRINA JURÍDICA: ¿QUÉ MÉTODO(S) PARA QUÉ TIPO DE DISCIPLINA?
}

\author{
Legal Doctrine: Which Method(s) \\ for What Kind of Discipline?
}

Mark VAN HOECKE ${ }^{2}$

\begin{abstract}
Sumario:
I. Desarrollos históricos II. ¿Qué tipo de disciplina es la doctrina jurídica? A) Una disciplina hermenéutica B) Una disciplina argumentativa C) Una disciplina empírica D) Una disciplina explicativa E) Una disciplina axiomática F) Una disciplina lógica G) Una disciplina normativa III. ¿Qué tipo de metodología para la investigación jurídica? A) Los datos empíricos en la investigación jurídica B) Formulación y comprobación de hipótesis C) La construcción de teorías en la doctrina jurídica IV. Conclusión V. Fuentes
\end{abstract}

Resumen: Este trabajo analiza el problema del estatuto de la doctrina jurídica. En primer lugar, se aborda la disciplina desde una perspectiva histórica, destacándose que, en sus orígenes, primaban sus perfiles argumentativos más que científicos. Posteriormente, examina cómo han influido en la misma los desarrollos de las ciencias positivas; es decir, si es posible que la doctrina jurídica sea o se equipare a una disciplina "cientifica". Una vez presentadas las distintas concepciones en las que la doctrina jurídica se ha presentado, el autor propone definirla como "disciplina empírico-hermenéutica". Sostiene que su núcleo sigue siendo la interpretación. Finalmente, incursiona en el tema de en qué medida la doctrina jurídica y su metodología se asemeja a ( $y$ diferencia de) otras disciplinas científicas para terminar proponiendo temas de debate abiertos a futuras investigaciones.

Palabras Clave: Doctrina Jurídica, Metodologías Jurídicas, Concepciones Jurídicas, Argumentación Jurídica, Interpretación Jurídica

Abstract: This paper analyses the status of legal doctrine. Starting by examining the historical aspects of the discipline, emphasis is put on its argumentation rather than its scientific characteristics. The author then asks whether the influence exerted by the developments of positive sciences over legal doctrine provide an adequate basis for the latter to be considered a "scientific" discipline in itself. Outlining the different conceptions of legal doctrine, the author proposes to define it as an "empirical-hermeneutic discipline". He claims that the notion of interpretation remains at its core. Finally, the author describes the similarities between legal

\footnotetext{
${ }^{1}$ Publicado en: M. Van Hoecke (ed.), Methodologies of Legal Research. Which Kind of Method for What Kind of Discipline?, Oxford-UK, Hart Publishing Ltd., 2011, pp. 1-18. Se traduce y publica con autorización expresa del autor. Traducción y Abstract de Francisco M. Mora Sifuentes, quien agradece a Gabriel M. Lentner y Daniël Overgaauw su generosa ayuda para las traducciones del alemán.

${ }^{2}$ Director de la Escuela de Doctorado en Artes, Humanidades y Derecho de la Universidad de Gent, Bélgica. Profesor Investigador en Teoría del Derecho y Derecho Comparado del Departamento de Derecho de la misma Universidad. Co-Director de la Academia Europea de Teoría del Derecho.
} 
doctrine, including its methodology, and other scientific disciplines. In conclusion, he outlines further topics for debate and further research.

Key Words: Legal Doctrine, Legal Metodologies, Legal Conceptions, Legal Argumentation, Legal Interpretation

\section{DESARROLLOS HISTÓRICOS}

La doctrina jurídica romana se desarrolló a partir el siglo II antes de Cristo, alcanzando un elevado nivel de sofisticación durante el siglo III después de Cristo. Su redescubrimiento y estudio renovado en la Bolonia del siglo XI marcó el inicio de la creación de las universidades. Durante toda la Edad Media la doctrina jurídica se consideraba una "disciplina científica”. En aquella época fue la “interpretación autorizada” y no la “investigación empírica”, el criterio principal para el estatus científico de una disciplina. Poco a poco esto fue cambiando. Sobre todo durante el siglo XVII. Pero a partir del siglo XIX el cambio fue drástico. El triunfo de las ciencias positivas alteró la concepción de la "ciencia" en las sociedades occidentales. La física se convirtió en el modelo a seguir. Una combinación de datos empíricos, las matemáticas, la verificación de hipótesis, el desarrollo de teorías de validez general -sin limitaciones geográficas- pasaron a ser el ideal para cualquier "disciplina académica". Sin embargo, ¿en qué parte de la doctrina jurídica estudiamos "datos empíricos", manejamos modelos matemáticos, comprobamos hipótesis o construimos teorías? El Derecho y la doctrina jurídica tienen claramente sus limitaciones geográficas. No tienen pretensión de "validez general" más allá de las fronteras geográficas del ordenamiento jurídico en cuestión.

A partir de mediados del siglo XIX, esas conclusiones han llevado repetidamente a la afirmación de que la "doctrina jurídica" pierde sus características básicas a fin de ser considerada "ciencia jurídica", mientras que, hasta ese momento, la doctrina jurídica se consideraba en gran medida como el modelo de "ciencia". ${ }^{3}$ Más recientemente, procedimientos de evaluación de la investigación y, especialmente, el reparto de recursos públicos entre "científicos" han puesto el tema en el centro de la discusión académica. Los juristas reaccionaron de distintas maneras a este tipo de presiones. La mayoría de ellos han destacado la utilidad práctica, o incluso la necesidad, de sus publicaciones para la práctica jurídica, enfatizando la importancia del Derecho en la sociedad. O, benignamente, han ignorado la crítica. Otros en cambio, la han aceptado, tomando el punto de vista empirista más estrecho de "ciencia",

\footnotetext{
3 En 1859, el teórico norteamericano David Dudley Finn escribió sobre la doctrina jurídica en los siguientes términos: "compara esta ciencia con cualquier otra; con aquellas de mayor extensión y de temas más exaltados. Toma incluso la astronomía, esa noble ciencia que pesa el sol y los planetas, mide sus distancias, traza sus órbitas y penetra en los secretos de las leyes que gobiernan sus movimientos. Sublime como esta ciencia, es solamente la ciencia de la materia inanimada, y de algunas leyes naturales; mientras que la ciencia que es el tema de nuestro discurso gobierna la acción de los seres humanos, inteligente e inmortal, penetra en los secretos de sus almas, somete sus voluntades, y se adapta a la variedad infinita de sus deseos, motivos y condiciones". Véase: FINN, David D., "Magnitude and Importance of Legal Science" (1859), ahora en: Presser, S. B., y Zainaldin, J. S. (eds.), Law and Jurisprudence in American History, Minnesota, West Publishing Co., 1995, p. 712. Desde una perspectiva distinta, Ivanhoe Tebaldeschi pudo, en 1979, argumentar que la doctrina jurídica es la disciplina más completa y, por lo tanto, el modelo de ciencia, puesto que combina razonamiento deductivo con razonamiento inductivo y pensamiento valorativo. TEBALDESCHI, I., Rechtswissenschaft als Modellwissenchaft, Viena, Springer Verlag, 1979, p. 156.
} 
buscando ajustar la investigación jurídica a ese modelo. ${ }^{4}$ En el siglo XIX este tipo de reacción alumbró la "teoría del Derecho" como "ciencia positiva del Derecho", una especie de Derecho natural empírico, una investigación de conceptos, reglas y principios jurídicos que la humanidad entera compartirías. Hubo alguna investigación en antropología social (Maine, Post), ${ }^{6}$ pero la misma se ha mantenido en gran medida como un programa de investigación; un programa olvidado desde la Primera Guerra Mundial. Tal y como he señalado, una reacción similar ocurrió a finales del siglo XIX y fundamentalmente durante el transcurso del siglo XX, con el nacimiento y desarrollo de otras disciplinas sociales enfocadas al Derecho: la sociología, la psicología jurídica o el análisis económico del Derecho. Todas estas disciplinas ofrecen investigación empírica y construyen teorías en cuestiones jurídicas. No obstante, estas últimas no pretenden reemplazar la doctrina jurídica. Únicamente buscan proveer de información valiosa de la realidad jurídica a los investigadores y operadores jurídicos así como a quienes elaboran políticas públicas. Desafortunadamente su impacto ha sido muy limitado. De tal forma que, actualmente, existe una situación esquizofrénica en la que mientras que una disciplina (la doctrina jurídica) básicamente estudia el Derecho como un sistema normativo, limitando sus "datos empíricos" a textos jurídicos y sentencias judiciales, otras disciplinas estudian la realidad jurídica, el Derecho como es. Los resultados de estas dos disciplinas y vertientes no se entrelazan de forma sistemática, ni se combinan o integran al nivel de la investigación jurídica.

Actualmente en muchos países la evaluación de la investigación y los recursos económicos asociados a ella han hecho aún más influyente el modo empírico de concebir la ciencia. Esto ha llegado hasta tal punto que ha provocado que algunos juristas y administradores públicos en las universidades piensen que la doctrina jurídica puede devenir en "científica" si se transforma en una ciencia social empírica (de Geest). En otras palabras: su propósito es poner fin a una tradición de más de dos milenios para imitar las ciencias empíricas que tienen un objetivo distinto. En lugar de concluir que la visión monista de ciencia, basada en la física, es errónea -o "falseable", utilizando su propia terminología- porque su esquema

\footnotetext{
4 "Welcher Abstand zeigt sich hier für die Jurisprudenz gegen die Naturwissenschaften": J. VON KIRCHMANN, Die Werthlosigkeit der Jurisprudenz als Wisseschaft, Berlin, Julius Springer, 1848, p. 14 [traducción propuesta: "Que distancia se muestra aquí para la jurisprudencia contra las ciencias naturales"]; VAN HOUTEN, S., Das Causalitätsgesetz in der Socialwissenschaft, Haarlem, HD Tjeenk Willink y Leipzig, FA Brockhaus, 1888, argumentando a favor del uso de los métodos de la física en la investigación jurídica, fundamentalmente focalizándose en las relaciones causales: "Der Grundstein der Socialwissenschaft, wie überhaupt aller Wissenschaft, its die volle, unbedingte Anerkennung des Causalitätsgesetzes" p. 5. [traducción propuesta: "El fundamento de las ciencias sociales, de hecho, como el de cualquier otra ciencia, es el reconocimiento incondicionado y total de la ley de la causalidad"]. Véase también: LUNDSTEDT, A. V. Die Unwissenschaftlichkeit der Rechtswissenschaft, Berlin, Grunewald, W Rothschild, vol. I., 1932; MULDER, T., Ik beschulding de rechtsgeleerde faculteit van onwetenschappelijkheid, Leiden, 1937; DE GEEST, G., "Hoe maken we van de rechtswetenschap een volwaardige wetenschap?, Nederlands Juristenblad, 2004, pp. 58-66.

5 Post, A. H., Einleitung in eine Naturwissenschaft des Rechts, Oldemburg, Verlag der Schulzchen Buchhandlug, 1872. Para una breve introducción a Post y a su investigación antropológico-jurídica, véase: LYALL, A., "Early German Legal Antropology: Albert Hermann Post and His Questionnaire", Journal of African Law, núm. 52, 2008, pp. 114-124 (con el cuestionario como apéndice en las pp. 124-138).

${ }^{6}$ WILKEN, G. A., De vrucht van de beoefening der ethnologie voor de vergelijkende rechtswetenschap, lección inaugural, Rijksuniversiteit Leiden (Leiden, EJ Brill, 1885); STEINMETZ, S. R., Ethnologische Studien zur ersten Entwicklung der Strafe, $2^{\mathrm{a}}$ ed., 2 vols., Groningen, P. Noordhoff, 1928, (la primera edición se publicó en Leiden en 1894); STEINMETZ, S. R., Rechtsverhältnisse von eingeborenen Völkern in Afrika und Ozeanien. Beantwortungen des Fragebogens der Internationalen Vereinigung für vergleichende Rechtswissenchaft und Volkswirtschaftslehre zu Berlin, Berlin, Julius Springer, 1903.
} 
no encaja con disciplinas tales como la doctrina jurídica, algunos han terminado señalando que la doctrina jurídica es (completamente) errónea, y que siempre lo ha sido. Este es un desarrollo peligroso, que, partiendo de premisas falsas (la unidad y la similitud de todas las disciplinas científicas), está poniendo en peligro el futuro de las ciencias humanísticas en general y de la doctrina jurídica en particular.

Por supuesto, las críticas a la doctrina jurídica están fundadas en parte: a menudo es demasiado descriptiva, demasiado autopoiética, no toma en cuenta el contexto del Derecho suficientemente; carece de una metodología clara y sus métodos suelen ser idénticos a aquellos de la práctica jurídica; es demasiado local, limitada a comunidades científicas muy pequeñas debido a la especialización y los límites geográficos; no hay mucha diferencia entre las publicaciones de los operadores jurídicos y las de los académicos. Todo lo anterior puede ser cierto, pero tal crítica, no es suficiente para desacreditar el carácter de disciplina que la doctrina jurídica tiene por mérito propio, con sus métodos apropiados.

En este trabajo voy a definir la doctrina jurídica como una "disciplina empírico-hermenéutica”. En efecto, tiene aspectos empíricos que la hacen perfectamente comparable con todas las disciplinas empíricas, pero el núcleo principal de la doctrina jurídica es la interpretación, y esto es algo que tiene en común con otras disciplinas (la teología o los estudios literarios).

Ahora bien: ¿cómo podemos describir la metodología de la doctrina jurídica con la terminología que utiliza la comunidad científica, sin limitarla o estrecharla de tal forma que pierda sus características esenciales?

En el curso de la historia, la doctrina jurídica se ha practicado y concebido de diferentes maneras, enfatizándose -en ocasiones demasiado- características divergentes de la misma. En lo que sigue, vamos a discutir los diferentes ángulos desde los cuales la doctrina jurídica se ha presentado y en qué medida éstos nos proporcionan una imagen fiel de la misma. Posteriormente analizaré la metodología de la investigación jurídica en términos de hipótesis y construcción de teorías.

\section{II. ¿QUÉ TIPO DE DISCIPLINA ES LA DOCTRINA JURÍDICA?}

\section{A) UNA DISCIPLINA HERMENÉUTICA}

Difícilmente puede negarse el hecho de que quienes se dedica a la investigación jurídica suelen interpretar textos y discutir qué interpretación elegir de entre varias divergentes. En este sentido la doctrina jurídica es una disciplina hermenéutica, de la misma forma que lo es, por ejemplo, el estudio de la literatura o de la historia -aunque esta última lo sea en menor medida-. La interpretación de textos ha estado en el centro de la doctrina jurídica desde sus comienzos en el Imperio Romano. ${ }^{7}$

En una disciplina hermenéutica los textos y documentos son el mayor objeto de investigación y su interpretación, según métodos estandarizados, es la actividad principal del investigador. Claramente tal es el caso de la doctrina jurídica.

A menudo la investigación jurídica se ha presentado como otro tipo de "ciencia" cuya dimensión hermenéutica se minimiza o, al menos, se le resta importancia. Esto ocurre, por

\footnotetext{
7 "L’oeuvre doctrinale, dans la tradition historique française et, plus largement, européene, est au premier chef d'interpétation de "lois" écrites... Et à cela ne s'est pas borné son rôle. Face à des sources diverses et hétérogènes, elle s'est trouvé aussi pour fonction d'unifier, de créer un ordre juridique cohérent et mème, à 
ejemplo, cuando los investigadores intentan distinguir la investigación jurídica de la práctica jurídica; o cuando separan la descripción del Derecho más claramente de su valoración; o bien, cuando la investigación jurídica se moldea conforme a las líneas metodológicas de otras disciplinas; y más específicamente, cuando se moldea mediante las ciencias positivas.

\section{B) UNA DISCIPLINA ARGUMENTATIVA}

Próxima a la concepción de la doctrina jurídica como disciplina hermenéutica está su concepción en tanto disciplina argumentativa. Lo que se enfatiza aquí es la argumentación para defender alguna interpretación o solución jurídica, más que la interpretación en sí misma. ${ }^{8}$ El enfoque argumentativo tiene la ventaja de situar las cosas en una perspectiva más amplia. Nos permite dar un paso atrás en el texto interpretado o cualquier otro documento. Una cuestión jurídica concreta puede responderse -o un caso resolverse- sobre la base de puntos de vista generalmente aceptados, o al menos aceptables. En la teoría de la argumentación tradicional esos puntos de vista se llaman "topoi". 9 En muchos casos las argumentaciones pueden respaldar alguna interpretación de uno o más textos, pero en otros casos la argumentación puede vagamente vincularse a esos textos. Así, por ejemplo, cuando se hace teniendo como base principios "implícitos" [unwritten legal principles], o cuando se colman lagunas en el Derecho, o cuando la literalidad de un texto se deja de lado en favor de un interés o valor que, para el caso en concreto, se considera más importante.

Desde la Edad Media hasta el siglo XVII la doctrina jurídica se desarrolló como una disciplina argumentativa que determinaba qué tipo de argumentos eran aceptables en qué casos a través de catálogos completos de argumentos. ${ }^{10}$ De hecho, interpretación y argumentación no pueden separarse la una de la otra; sea en la práctica jurídica o en la doctrina. Cada texto interpretado necesita de argumentos cuando interpretaciones divergentes pueden defenderse razonablemente y la argumentación jurídica, a su vez, siempre debe defenderse teniendo

partir du XVIème siècle, systématique, préparant ainsi les voies de la codification”. Véase: THIERAU, J.-L., "La doctrine civiliste avant le Code civil" en: Y. Poirmeur , et al., La doctrine juridique, Paris, Presses Universitaires de France, 1993, pp. 13-51, aquí p. 16 y ss. [traducción: "La obra doctrinal en la tradición histórica francesa, y en general la europea, se dedica primordialmente a la interpretación de 'leyes' escritas... Y ahí no queda su función. Ante fuentes diversas y homogéneas, también se dio a la tarea de unificar, de crear un orden jurídico coherente así como, desde el siglo XVI, sistemático, allanando el camino de la codificación”].

${ }^{8}$ La teoría de la argumentación jurídica siempre ha estado en el núcleo de los escritos en teoría jurídica. Esta es la razón por la cual la concepción de la doctrina jurídica en tanto disciplina argumentativa a menudo actúa como su fundamento implícito (véase, por ejemplo: PERELMAN, CH., Logique juridique. Nouvelle rhétorique, Paris, Dalloz, 1976; ALEXY, R., Theorie der Juristischen Argumentation, Suhrkamp Verlag, Frankfurt am Main, 1983; DWORKIN, R., Law's Empire, London, Fontana Press, 1986, pp. 13-14, donde se sostiene esto de forma explícita [N. T.: El profesor M. Van Hoecke, autor del artículo, me pide que añada a esta referencia que el lector en lengua castellana puede acudir al trabajo de: ATIENZA, M., El derecho como argumentación, Barcelona, Ariel, 2006]. En algunas ocasiones esta concepción de la doctrina jurídica como disciplina argumentativa fue defendida de forma explícita: SMITS, J., "Redefining Normative Legal Science: Towards an Argumentantive Discipline” en: F. Grünfeld et al y F. Commands (eds)., Methods of Human Rights Research, Antwerp, Intersentia, 2009; SMITH, C., et al., "Criteria voor goed rechtswetenschappelijk onderzoek”, Nederlands Juristenblad, 2008, pp. 685-690, en la p. 690, donde, siguiendo a Ronald Dworkin, el trabajo del investigador y del juez más bien se denomina como una "práctica argumentativa" en lugar de una “disciplina normativa”.

${ }^{9}$ VIEHWEG, Th., Topik und Jurisprudenz: ein Beitragzur Rechtswissenschaftlichen Grundlagenforschung, $5^{\mathrm{a}}$. ed. München, Beck Verlag 1974; STRUCK, G., Topische Jurisprudenz, Frankfurt, Athenäun Verlag, 1971.

${ }^{10}$ VAN DEN BERGH, G.C.C.J., Geleerd recht. Een geschiedenis van de Europese rechtswetenschap in vogelvlucht, $2^{\text {a }}$. ed., Deventer, Kluwer, 1985, p. 6. 
como base textos interpretados. Es decir, tanto la doctrina como la práctica jurídica son hermenéuticas y argumentativas, de tal forma que argumentación e interpretación se presentan en gran medida como las dos caras de la misma actividad. La interpretación es el fin y la argumentación el medio para sustentar esa interpretación. Por lo tanto, si tuviera que elegir la etiqueta más apropiada para la doctrina jurídica diría que ésta es una "disciplina hermenéutica" más que argumentativa.

\section{C) UNA DISCIPLINA EMPÍRICA}

Como señalé anteriormente, desde el siglo XIX y bajo el influjo del éxito de las ciencias positivas ha habido intentos de desarrollar la doctrina jurídica como una disciplina empírica. ${ }^{11}$ Esta pretensión la asumió explícitamente Alf Ross:

La interpretación del estudio doctrinal del Derecho presentado en este libro se basa en el postulado de que el principio de verificación debe aplicarse también a este campo de conocimiento- que el estudio doctrinal del Derecho debe reconocerse como una ciencia social empírica. ${ }^{12}$

Según Ross, esa verificación empírica tiene lugar a través de la comprobación de las proposiciones de la doctrina jurídica confrontadas con la práctica judicial: "mi interpretación, basada en los análisis anteriores, es que el contenido real de las proposiciones doctrinales hacen referencia a la acción de los tribunales bajo ciertas condiciones". ${ }^{13}$

Este punto de vista es típico de los movimientos realistas. Ross fue el último gran representante del realismo escandinavo, pero en este punto estaba más próximo al realismo norteamericano: "Las profecías de lo que los jueces efectivamente harán, y nada más pretensioso, es lo que yo entiendo por Derecho", según la famosa formulación de Oliver Wendell Holmes. ${ }^{14}$ La concepción de la doctrina jurídica de Ross como disciplina empírica concuerda únicamente con este tipo de aproximación "realista”. En la actualidad, el realismo jurídico no es muy popular en Europa; tampoco en los países escandinavos. Por tanto, y en sí misma, esta concepción de la doctrina jurídica no puede considerarse un modelo viable.

Otros han argumentado que el fenómeno que los estudiosos del Derecho analizan y observan es, de hecho, su información empírica, que equivale a "normas jurídicas". ${ }^{15}$ Sin embargo, lo que es cuestionable es que las normas jurídicas puedan observarse empíricamente. Y si tal tarea no es posible, ¿dónde y cómo las encontramos? Para Gerrit De Geest las mismas se encuentran a través de la lectura de decisiones judiciales. Esta forma de ver las cosas sugiere que esas normas jurídicas existen únicamente en la medida en que han sido aplicadas por los

\footnotetext{
${ }^{11}$ No se trató de una pretensión exclusiva de la doctrina jurídica: "Ocasionalmente, los investigadores en 'disciplinas inferiores' que aspiran al estatus de los científicos naturales han intentado importar el método 'científico' o empírico a su trabajo. En el siglo XIX, por ejemplo, esos esfuerzos redefinieron de las disciplinas científicas, dándoles muchas de sus características distintivas en la actualidad". Véase: VICK, D. W., "Interdisciplinarity and the Discipline of Law", Journal of Law and Society, núm. 31, 2004, pp. 163-193, aquí p. 172.

${ }^{12}$ ROSS, A., On Law and Justice, London, Stevens \& Sons, 1958, p. 40. Esta visión fue explícitamente rechaza en: SAMUEL, G., "Is Law Really a Social Science? A View from Comparative Law", Cambridge Law Journal, núm. 67, 2008, pp. 288-312, entre otros lugares en la p. 319.

${ }^{13}$ ROSS, A., On Law and Justice, cit.

${ }^{14}$ HOLMES, O. W., "The Path of the Law", Harvard Law Review, núm. 10, 1897, pp. 457-478, aquí p. 461.

${ }^{15}$ DE GEEST., G., "Hoe maken we van de rechtswetenschap een volwaardige wetenshap?", Nederlands Juristenblad, 2004, pp. 58-66, aquí p. 69.
} 
jueces. Con ello De Geest está siguiendo a Alf Ross y al realismo norteamericano. No obstante, De Geest se contradice a sí mismo en parte cuando define la "verdad empírica" en la interpretación del Derecho como "lo que el legislador o el juez quisieron decir realmente". ${ }^{16}$ Como métodos utilizados en ese contexto, menciona los siguientes:

a) el análisis de textos;

b) la lógica (por ejemplo, el silogismo)

c) el trabajo de campo (entrevistas incluidas)

d) las estadísticas, $y$

e) métodos de investigación histórica. ${ }^{17}$

Es interesante advertir que ningún método psicológico se menciona como posible medio para descubrir lo que el juez o el legislador "quisieron decir realmente". Sin entrar a discutir más la posición de De Geest aquí, es obvio que su etiqueta "investigación empírica” cubre una gran variedad de elementos. Ello mostraría (según el propio De Geest) que la doctrina jurídica tiene partes de una disciplina hermenéutica (análisis textual), pero también de una axiomática (lógica) e histórica. De hecho, no podría reducirse a un tipo único de disciplina, sino que la doctrina jurídica sería una combinación de todas ellas. Por supuesto que algunas entre las anteriores podrían considerarse más importantes, más decisivas o más típicas que otras; pero, a diferencia de otras disciplinas, como por ejemplo las matemáticas, no estamos ante una disciplina unidimensional.

Para Hans Albert, el objeto de una doctrina jurídica empírica es más amplio que únicamente el análisis de normas jurídicas. La misma incluye también la influencia que ejercen dichas normas sobre los miembros de la sociedad en cuestión. ${ }^{18}$ Ello da como resultado una combinación entre la doctrina jurídica tradicional y la sociología jurídica. Existen razones de peso para este tipo de planteamiento, pero, en la mayoría de los casos, parece muy difícil llevarlo a la práctica. Es más, se tendrían que incluir otras disciplinas tales como la economía, la psicología u otras similares. Sin embargo, al incluirlas en la doctrina jurídica surgen muchas más cuestiones además de la de su factibilidad.

Para la Escuela Histórica del Derecho alemana ${ }^{19}$ del siglo XIX y para un movimiento equiparable en los Estados Unidos durante el mismo periodo, ${ }^{20}$ los elementos históricos constituían la información empírica más importante:

"El hombre ha de estudiarse en cada periodo de su existencia social, desde el estado salvaje hasta el Estado civilizado, con la finalidad de percibir la gran verdad: que en cada

\footnotetext{
${ }^{16}$ Ibid. p. 59 .

${ }^{17}$ Ibid. p. 61.

${ }^{18}$ ALBERT, H., “Kennis en Recht” en: F. D. Heyt (ed.), Rationaliteit in wetenschap en samenleving, Alphen ann de Rijn, Samsom, 1976, p. 183.

${ }^{19}$ Véase: BROCKMÖLLER, A., Die Entstehung der Rechtstheorie im 19. Jahrhundert in Deutschland, BadenBaden, Nomos Verlag, 1997, especialmente p. 64 y ss. (sobre Hugo) y p. 83 y ss. (sobre Savigny).

${ }^{20}$ Véase: SCHWEBER, H., "Law and the Natural Sciences in Nineteenth-Century American Universities", en: S. S. Silbey (ed.), Law and Science, The International Library of Essays in Law and Society, Aldershot, Ashgate, 2008, pp. 3-23.
} 
condición de libertad, de inteligencia, de comercio y de riqueza, sus hábitos, sus virtudes, sus vicios, sus objetos de sus deseos y, por tanto, las leyes necesarias para su gobierno, son esencialmente las mismas". ${ }^{21}$

Esta aproximación claramente representa la creencia en una suerte de "Derecho natural" que puede extraerse empíricamente. Tal idea solía ser muy popular en Europa y en los Estados Unidos durante el siglo XIX, pero hoy en día ha sido abandonada completamente.

Para otros, el objeto de la investigación empírica son datos sociológicos, económicos o socio-psicológicos o, de modo más general, el "comportamiento humano". ${ }^{22}$

La investigación empírica es mucho más útil en disciplinas como la física, donde la realidad se estudia de forma independiente a dicha disciplina. En disciplinas como las matemáticas o la teología, la investigación empírica no parece demasiado relevante. Los modelos matemáticos o las visiones teológicas crean su propia realidad, la cual, por definición, no puede verificarse empíricamente. En gran medida, lo mismo vale también para la doctrina jurídica. Si bien es cierto que una norma "existe" y que su existencia puede verificarse empíricamente, en cualquier caso, la doctrina jurídica trata fundamentalmente de la interpretación de esa norma o de su ponderación con otras normas o principios jurídicos.

Las interpretaciones se sostienen con argumentos y esos argumentos pueden referirse parcialmente a una realidad "objetiva”. En este sentido, la corrección de un argumento puede comprobarse empíricamente. No obstante, la mayoría de los argumentos en el razonamiento jurídico no son "verdaderos" o "falsos" sino convincentes en mayor o menor medida. Los mismos no están cualificados para su verificación empírica.

\section{D) UNA DISCIPLINA EXPLICATIVA}

Una cuarta concepción de doctrina jurídica la considera una disciplina explicativa. Según este punto de vista, la doctrina jurídica explica por qué una regla es una norma jurídica válida en una sociedad determinada. Esta explicación puede ser histórica, sociológica, psicológica, económica u otra similar, pero también puede estar basada en una lógica interna. En esta aproximación, la existencia de una regla puede "explicarse" por la existencia de una norma superior, de la cual se deriva dicha regla; ${ }^{23}$ o por la existencia de valores o principios subyacentes o mediante una red más amplia de reglas y principios jurídicos.

El primer tipo de explicación reduce la doctrina jurídica a una o más de las ciencias sociales mencionadas. La segunda "explicación" -la interna- reduce este concepto a algo que ya no forma parte importante de la actividad jurídica académica, que no encaja en nuestra comprensión común de lo que significa "explicar" algo. Como señaló Aleksander Peczenik, estamos ante una estrategia velada en la que la presentación de la doctrina jurídica como disciplina "explicativa" nos permitiría considerarla como una "ciencia objetiva"; dicha estrategia también facilita ocultar la legitimación de una regla detrás de la fachada de la propia

\footnotetext{
${ }^{21}$ GREENLEAF, S., A Discourse Pronounced at the Inauguration of the Authors as Royall Professor of Law in Harvard University, Cambridge, Massachusetts, James Munroe, 1834, aquí p. 14.

${ }^{22}$ LOTH, M. A., "Regel-geleid gedrag; over het object van empirische rechtswetenschap", Rechtsfilosofie \& Rechtstheorie. Netherlands Journal for Legal Philosophy and Jurisprudence, núm. 3, 1983, pp. 213-228, aquí p. 213 .

${ }^{23}$ VAN QUICKENBORNE, M., "Rechsstudie als wetenschap" en: Actori incubit probatio, Antwerp, Kluwer, 1975 , p. 223.
} 
"explicación" . ${ }^{24}$ De hecho, aquí no se "explica" nada. Más bien, se postulan unos valores o principios, o se aduce la interpretación de una norma superior, la cual, debe legitimar la regla que uno hace derivar de aquellos.

Otros puntos de vista consideran que el objeto de la investigación jurídica es la explicación del comportamiento humano basado en reglas, ${ }^{25}$ en interacción con otros actores. ${ }^{26} \mathrm{Sin}$ embargo, este objetivo es más propio de la sociología jurídica que de la doctrina jurídica. Aquí, en este enfoque, la actividad del investigador jurídico deviene en "tecnología social". ${ }^{27}$

La explicación de los porqués y los paraqués de los conceptos jurídicos, de las reglas, los principios o sus interpretaciones no puede considerarse algo carente de importancia en la investigación jurídica. Al contrario, lo anterior resulta indispensable para su correcta comprensión. En todo caso, debe señalarse que la explicación no es el principal objetivo de la investigación en materia jurídica -a excepción, quizás, de la época del seguimiento de normas a través de las grandes codificaciones-. Las actividades de investigación hermenéutica y las explicativas están estrechamente vinculadas entre sí, es la explicación la que está al servicio de la interpretación, no al revés. Por lo tanto, no podemos concluir que la doctrina jurídica sea primordialmente una disciplina explicativa.

\section{E) UNA DISCIPLINA AXIOMÁTICA}

En algunas ocasiones la doctrina jurídica ha sido vista en Europa continental como una disciplina axiomática, tal y como las matemáticas. Gustav Hugo, uno de los fundadores de la Escuela Histórica del Derecho alemana del siglo XVII, la concebía de la siguiente forma:

Jurisprudenz und Mathematik grenzen auch näher an einander als mancher, der weder noch Mathematiker ist, weiss... Auch die Jurisprudenz beruht in der Theorie auf einer Art von Construction und auch die Jurisprudenz ist in der Theorie eine exacte Wissenschaft. ${ }^{28}$

La doctrina jurídica para Hugo era una ciencia aplicada exacta, aunque dotada también de cierta dimensión empírica. Esta forma de concebir la doctrina jurídica culminaría en Alemania a finales del siglo XVII en el movimiento denominado "Jurisprudencia de Con-

\footnotetext{
${ }^{24}$ PECZENIK, A., "Scientia Juris. Legal Doctrine as Knowledge of Law and as a Source of Law", en: E. Pattaro (ed.), A Treatise of Legal Philosophy and General Jurisprudence, Dordrecht, Springer, 2005, vol. 4, p. 4.

25 "Wer den Sinn des Gesetzes bestimmen möchte, muss sich eo ipso Gedanken über die mit ihm intendierten Wirkungen und die damit angestrebte Ordnung machen. Solche Überlegungen machen die Verwendung nomologischen Wissens erforderlich, denn die Steuerungswirkungen von Gesetzen und Auslegungen sind nicht einfach logische Konsequenzen der betreffenden Aussagen”. ALBERT, H., Traktat über rationale Praxis, Tübingen, J. C. B Mohr, 1978, pp. 79-80. [traducción propuesta: "Cualquiera que trata de determinar el significado de una ley debe eo ipso hacer una reflexión sobre el efecto deseado y el fin que persigue. Tales consideraciones requieren el uso del conocimiento nomológico, ya que el efecto de control de las leyes y la interpretación no son consecuencias lógicas simples de las proposiciones respectivas"].

${ }^{26}$ LOTH, M. A., "Regel-geleid gedrag; over het object van empirische rechtswetenschap", cit., aquí p. 221.

${ }^{27}$ Ibid. p. 225.

${ }^{28}$ HUGO, G., Lehrbuch eines civilistischen Cursus, Philosophische Encyclopedie für Juristen, Berlin, 1799, vol. 5, p. 10, parágrafo 8. [traducción propuesta: "La jurisprudencia y las fronteras matemáticas están entre sí más cerca de lo que quienes no son ni juristas ni matemáticos, saben... también la jurisprudencia se basa en la teoría de algún tipo de construcción y la jurisprudencia es, en teoría, una ciencia exacta"].
} 
ceptos" [Begriffsjurisprudenz], quienes concebían al Derecho como una suerte de álgebra de los conceptos jurídicos.

En la segunda mitad del siglo XX este enfoque tuvo un resurgimiento. Dicho resurgimiento, no obstante, fue muy corto. Algunos de sus cultivadores más optimistas tenían la esperanza de abarcar todo el Derecho en una lógica formal y/o en programas informáticos. Sin embargo sus empeños, hasta ahora, no han tenido mucho éxito.

\section{F) UNA DISCIPLINA LÓGICA}

La perspectiva de la doctrina jurídica como una disciplina lógica es, en algún sentido, una versión más moderada del modelo puramente axiomático:

In der Rechtsordnung spielt das Logische nur eine sekundäre Rolle neben den alogischen Momenten. In der Rechtsanwendung spielen diese alogische. Momente die sekundäre Rolle neben dem Logischen. In der Rechtswissenschaft endlich herrschen ausschliesslich die logische Funktionen: die Jurisprudenz dient mit logischen Mitteln dem logischen Zwecke der Systematisierung. ${ }^{29}$

Incluso si el Derecho no siempre es lógico en la práctica, para Julius Moor la doctrina jurídica debe ser exclusivamente lógica en cuanto a la forma en que sistematizamos el Derecho. La mayoría de los estudiosos del Derecho, con visiones distintas sobre la doctrina jurídica como disciplina, también han enfatizado la importancia de la lógica tanto en el razonamiento jurídico como en la presentación científica del material jurídico. Sin embargo, (los contenidos de) los materiales normativos también son muy indefinidos como para posibilitar que concibamos la doctrina jurídica como una disciplina puramente lógica. Mucho depende de la interpretación de principios, reglas y conceptos jurídicos. Por lo tanto, incluso siendo la lógica muy importante en el Derecho y en la investigación jurídica, debe señalarse que la interpretación es aún más importante. Sea como fuere, la coherencia lógica es una característica de la investigación científica en cualquier disciplina y no es sólo una característica típica de las ciencias jurídicas.

\section{G) UNA DISCIPLINA NORMATIVA}

La doctrina jurídica a menudo se denomina una disciplina normativa, que no sólo describe y sistematiza normas (es decir, una disciplina sobre normas) sino que, en gran medida, es una disciplina que toma o asume posiciones normativas; que hace elecciones entre valores e intereses. Esto, de hecho, resulta inevitable cuando, por ejemplo, se prefiere una interpretación sobre otras alternativas dadas. En última instancia, esa elección se adopta al otorgar mayor peso a algunos valores o intereses determinados en detrimento de otros que también

\footnotetext{
${ }^{29}$ MOÓR, J. "Das Logische im Recht”, Revue Internationale de la Théorie du Droit/Internationale Zeitschrift für Theorie des Rechts, 1927-1928, pp. 157-203, aquí p. 203 [traducción propuesta: "En un sistema u ordenamiento jurídico, tanto la lógica como los elementos ajenos a la lógica juegan un papel secundario. En la aplicación del derecho se utilizan ambos elementos, tanto los lógicos como los ajenos a la lógica. Ahora bien, en la jurisprudencia, sólo predomina la lógica: la jurisprudencia para cumplir con el "propósito lógico", sistematiza valiéndose de medios lógicos”]. Véase también, aunque de forma menos explícita: KRANENBURG, R., De Grondslagen der Rechtswetenschap. Jurdische kennisleer en methodologie, $5^{\mathrm{a}}$ ed., Harleem, HD Tjeenk Willink, 1955, p. 30 y ss.
} 
están en juego. Para otros, la doctrina jurídica es fundamentalmente la búsqueda de "un Derecho mejor" ${ }^{30}$ Esta posición hace referencia a elementos que son externos al Derecho y a la doctrina jurídica: la filosofía, la moral, la historia, la sociología, la economía o la política. Por consiguiente, la búsqueda de "un Derecho mejor" puede requerir investigación empírica; especialmente, cuando "mejor" significa mejor desde un punto de vista económico o sociológico o cuando trata de hacer referencia a las "convicciones morales (o políticas) prevalecientes".

Este enfoque normativo corre el riesgo de caer en la subjetividad. Sobre todo, cuando el investigador intenta presentar puntos de vista personales o sus propias convicciones como si fueran "el Derecho". Debería resultar obvio que un enfoque normativo de este tipo sólo podría tener estatus científico si el mismo busca un consenso intersubjetivo de la opinión que prevalece entre los estudiosos del Derecho o entre los juristas en general (especialmente, de jueces y académicos que han hecho públicos sus puntos de vista por medio de decisiones judiciales o de otro tipo de publicaciones). Por lo demás, se puede comprobar empíricamente si una opción es la que (en gran medida) prevalece entre los profesionales del Derecho o en una sociedad determinada.

Para Hans Kelsen que la doctrina jurídica sea una disciplina normativa es una cuestión de lógica interna, que no está vinculada a ningún criterio externo al propio Derecho que lo haga "mejor". Para él, la distinción entre "disciplinas descriptivas" y "disciplinas normativas" es una división básica entre las ciencias. Las disciplinas descriptivas -como las ciencias exactas-, buscan relaciones causales; mientras que las disciplinas normativas -como la doctrina jurídica o la ética-, utilizan la "imputación" como método. ${ }^{31}$ La "imputación" significa la determinación de la existencia de alguna obligación (en un sentido amplio) y/o el incumplimiento de la misma. Esa obligación se deriva, por medio de una lógica jurídica interna, de los propios elementos del sistema jurídico. Ahora bien, Kelsen subestima notablemente la importancia de la interpretación en el Derecho, así como la influencia de elementos extra-jurídicos en dicha interpretación. La razón principal de esta visión poco realista es su teoría del "significado", que Kelsen reduce al significado psicológico del emisor; es decir, a la intención de aquellos que han emitido una regla o un mandato. ${ }^{32}$ Con esa premisa puede minimizar el elemento hermenéutico en la investigación jurídica y enfatizar el carácter normativo del Derecho.

\section{III. ¿QUÉ TIPO DE METODOLOGÍA PARA LA INVESTIGACIÓN JURÍDICA?}

Llegados a este punto debería resultar claro ya que distintas concepciones de la doctrina jurídica implican metodologías muy diferentes. Ahora bien, si aceptamos que la doctrina jurídica es fundamentalmente una disciplina hermenéutica -que encaja mejor en la manera

\footnotetext{
30 WENDT, J. A. I., De methode der rechtswetenschap vanuit kritisch-rationeel perspectief, Zutphen, París, 2008, p. 141.

${ }^{31}$ KELSEN, H., General Theory of Norms, Oxford, Clarendon Press, 1991, pp. 22-25.

32 "Alguien que emite un mandato pretende algo. Espera que la otra persona entienda esa pretensión. Mediante su mandato pretende que esa otra persona se comporte de determinada manera. Ese es el significado de su acto o voluntad" (ibid. p. 32). Por lo tanto, según Kelsen es más correcto decir que "una norma es un significado", que a decir que "una norma tiene un significado". Este punto de vista es completamente insostenible. Véanse mis comentarios sobre este punto en particular en: VAN HOECKE, M., Law as Communication, Oxford, Hart Publishing, 2002, pp. 128-130.
} 
en que la doctrina jurídica ha sido concebida durante la mayor parte del tiempo en la mayoría de los sistemas jurídicos- podemos describir su metodología de la siguiente manera.

Los investigadores jurídicos recaban datos empíricos (legislación, sentencias, casos relevantes), formulan hipótesis sobre su sentido y alcance que, a continuación, ponen a prueba utilizando los cánones clásicos de interpretación. En una etapa posterior, construyen teorías (v. gr., acerca de la fuerza vinculante directa del Derecho de la Unión Europea) que ponen a prueba y de las que derivan nuevas hipótesis (v. gr., sobre la validez, sentido y alcance de una regla doméstica que entra en conflicto con el Derecho de la Unión). Descrita de este modo, la doctrina jurídica encaja perfectamente con la metodología de otras disciplinas: "La investigación científica, vista en una perspectiva muy amplia, tiene dos facetas principales. La primera es la verificación y el descubrimiento de hechos; la segunda, es la construcción de hipótesis y teorías".33

\section{A. LOS DATOS EMPÍRICOS UTILIZADOS EN LA DOCTRINA JURÍDICA}

En una primera etapa, la doctrina jurídica recaba todo el material relevante, en particular:

a) Fuentes normativas, tales como textos legislativos, tratados, principios generales del Derecho, derecho consuetudinario (costumbre), precedentes vinculantes, y otros similares; $y$

b) Fuentes autorizadas fiables, tales como sentencias, que no constituyan precedente vinculante y trabajos académicos sobre la materia.

La última categoría tiene una posición ambivalente, puesto que no se trata de algo externo a la propia doctrina jurídica, incluso en el caso de que sea externo en términos generales al investigador individualmente considerado. Aquí nos encontramos, por un lado, con una combinación de artículos doctrinales académicos como fuentes autorizadas de Derecho y, por otro, con la doctrina jurídica a modo de comunidad científica que debate, acepta o rechaza las posiciones que toman sus colegas así como las teorías que ellos proponen.

Generalmente, la discusión sobre las fuentes del Derecho es a propósito de su relevancia. Por ejemplo, si una ley ha sido declarada inconstitucional por el Tribunal Constitucional la misma deviene irrelevante dentro del material empírico con el que el investigador jurídico construirá su hipótesis o sus teorías. Un precedente vinculante será más relevante que uno que no lo sea. De la misma forma, una decisión (no vinculante) de la Corte Suprema será más relevante que una que haya sido tomada por una corte inferior, etc.

Una publicación de un profesor que con carácter general sea reconocido como una autoridad en la materia tendrá mayor peso que la primera publicación de un joven académico. Una argumentación bien sustentada será más relevante que el simple posicionamiento a favor de un punto de vista cuando esto último se hace sin argumentaciones ulteriores.

En el caso de fuentes normativas, la relevancia, en primera instancia, será una cuestión de validez: ¿en la actualidad esta regla forma parte de nuestro sistema jurídico o no? Se trata de una elección binaria entre su validez o invalidez.

En el plano de la interpretación, sin embargo, la relevancia deviene en una cuestión de grado: al ponderar y balancear la fuerza normativa de reglas y principios igualmente válidos,

\footnotetext{
${ }^{33}$ VON WRIGHT, G. H., Explanation and Understanding, Ithaca, Cornell University Press, 1971, p. 1. 
puede que uno sea considerado más relevante que otro, aun cuando esa mayor relevancia sólo se de en el caso concreto.

La relevancia de las fuentes autorizadas será siempre una cuestión de grado. Incluso el profesor más aclamado puede tener un momento de debilidad mientras que la primera publicación de un joven académico prometedor puede ser brillante. En algunas ocasiones las decisiones de la Corte Suprema son ampliamente rechazadas tanto por tribunales inferiores como por la doctrina jurídica y, por lo tanto, pierden gran parte de su relevancia. Las decisiones tomadas - por ejemplo por la Corte Suprema de Estados Unidos- por una mayoría de cinco jueces sobre cuatro será menos autorizada que una tomada por unanimidad.

En cualquier caso, todas esas fuentes tienen al menos algo de relevancia y el investigador o la investigadora deberá tenerlas en cuenta en su trabajo.

Un punto delicado es la representatividad de las sentencias publicadas. Las sentencias judiciales que realmente se publican representan únicamente un pequeño porcentaje de todas las sentencias que se dictan (en Bélgica apenas el uno o dos porciento). Sin embargo, un gran porcentaje se está haciendo accesible por medio de Internet. Es cierto que, en muchas ocasiones, en la decisión sobre qué sentencias publicar pesan más los motivos circunstanciales, anecdóticos, que los científicos. ${ }^{34}$ Incluso no todas las decisiones de las cortes supremas se publican. El almacenamiento electrónico adecuado de todas las decisiones judiciales en programas y bases de datos apropiados debería permitir un estudio sistemático de todas las decisiones judiciales tomadas en alguna materia o en algún problema jurídico específico, incluyendo, por supuesto, el análisis estadístico. Sin embargo, cabe resaltar que, a día de hoy, esto todavía no es posible ni operativo en muchos sistemas jurídicos.

\section{B) FORMULACIÓN Y COMPROBACIÓN DE HIPÓTESIS DE INVESTIGACIÓN}

Cualquier tipo de investigación científica comienza a partir de un problema, de alguna pregunta o serie de preguntas. En algunas ocasiones, una simple observación de hechos conduce de manera casi espontánea a una pregunta de investigación. Así, por ejemplo, cuando existen dos puntos de vista contradictorios dentro de la jurisprudencia y la doctrina jurídica, o entre jueces superiores e inferiores, o entre la jurisprudencia por un lado y la doctrina jurídica por el otro el investigador buscará automáticamente una explicación para esas posturas divergentes. Buscará también argumentos que le permitan decantarse definitivamente a favor de una de ellas o que, en otros casos, permitirían arribar a una tercera alternativa más convincente.

En otros casos, la pregunta de investigación será formulada sobre la base de la observación previa en otro contexto y los datos empíricos serán seleccionados conscientemente a la vista de la pregunta de investigación. Lo anterior no es algo que concierne sólo al Derecho sino que es común a todas las disciplinas: "Es claro, por tanto, que deben seleccionarse los hecho que, en base a las hipótesis, sean relevantes para resolver un problema dado". 35

Si por ejemplo queremos averiguar hasta qué punto algunas áreas del Derecho Privado -en algunos sistemas jurídicos continentales europeos- pueden reorganizarse inspirándose

\footnotetext{
${ }^{34}$ Por ejemplo, una demanda para obtener el derecho a visitar el perro propio después de un divorcio, cuando la ex pareja se quedó con el animal.

${ }^{35}$ NAGEL, E., “The Nature and Aim of Science”, en: S. Morgenbesser (ed.), Philosophy of Science Today, New York, Basic Books, 1967, pp. 3-13, aquí p. 10.
} 
en el concepto inglés de "trust", uno probablemente recabará datos relativos al Derecho concursal pero no de todas las áreas del Derecho privado.

Sea como fuere, la observación "científica" no es una percepción neutral de unos hechos que se nos presentan de manera espontánea. Siempre nos encontramos frente a una lectura específica de hechos seleccionados, dirigidos por la pregunta de investigación. La lectura de una visión puramente descriptiva de los precedentes judiciales en un espacio determinado campo y periodo puede conducir a la formulación de un problema jurídico, en vista del cual se recabará material adicional.

Como sucede en toda disciplina, la observación de los datos empíricos posee una orientación teórica. Un problema se formula dentro de algún marco teórico. Además del propósito de resolver un problema, los resultados de una investigación pueden también confirmar, refinar o falsear esos presupuestos teoréticos. La selección de los datos jurídicos relevantes debe hacerse sobre la base de una teoría de las fuentes jurídicas: ¿qué fuentes jurídicas son relevantes hoy en día en este sistema jurídico, y cuál es la relación jerárquica entre ellas? Esto puede, por supuesto, llevarnos a algunas preguntas de investigación del tipo: “¿el Derecho Europeo tiene prioridad sobre el orden constitucional nacional?"

Ni la selección de datos empíricos ni su descripción son actividades neutrales. Cuando el American Law Institute comenzó, en 1923, los “Restatements" del Derecho consuetudinario americano, se presentaban como una actividad neutral y apolítica. El Instituto fue duramente criticado porque explícitamente partió de las premisas falsas de "que es posible describir el Derecho tal y como es en términos neutrales" (sacando a relucir la interrelación entre descripción e interpretación), "que es posible hacer proposiciones significativas de las reglas jurídicas sin referencia a su justificación” (la finalidad del Derecho en su contexto interpretativo), o "sin referencia alguna al contexto práctico de su operación" (aplicación concreta como marco o contexto de interpretación"). ${ }^{36}$

De hecho, cuando formulamos normas jurídicas, tal y como aparecen en las fuentes jurídicas válidas, los textos que suscitan alguna cuestión se interpretan. Normalmente, no existen problemas de interpretación porque hay un consenso implícito sobre el significado preciso del texto, pero en ocasiones nos topamos con diversas lecturas del mismo texto, o con que el investigador tiene que determinar el alcance y significado exacto de una nueva norma promulgada o de una decisión reciente de algún tribunal. En tales casos, está claro que el académico está formulando una hipótesis sobre la validez y el significado preciso de ese texto jurídicamente relevante (relevante dentro del sistema jurídico dado en el momento de la investigación) ${ }^{37}$. En otras palabras, la interpretación está en el núcleo de toda la actividad que se realiza en la investigación jurídica. Las preguntas de investigación en la doctrina jurídica

\footnotetext{
${ }^{36}$ Véase sobre este punto: TWINING, W., Blackstone's Tower: The English Law School, London, Sweet \& Maxwell, 1994, p. 134.

${ }^{37}$ Véase, por ejemplo: ANDRÉ, A., "Was heisst rechtswissenschafliche Forschung?", Juristenzeitung, 1970, pp. 396-401, aquí p. 400; ALBERT, H., Traktak über rationale Praxis, cit., p. 80; AARNIO, A., Philosophical Perspectives in Jurisprudence, Acta Philosophica Fennica, Helsinsky, Academic Bookstore, 1983, pp. 163-184 (sobre la verdad y validez de las proposiciones interpretativas en la dogmática jurídica). En cambio: Vistos desde la perspectiva del tiempo, todas las proposiciones jurídicas, sean del legislador, de los jueces, o de los juristas, no son más que hipótesis de trabajo". GOFF OF CHIEVELY, Lord R. L. A., "Judge, Jurist and Legislature”, Denning Law Journal, núm. 2, 1987, pp. 79-95, aquí p. 8 o.
} 
están, de hecho, muy vinculadas al preciso significado y alcance de los conceptos jurídicos, reglas jurídicas, principios jurídicos y/o construcciones jurídicas.

Cada descripción del Derecho incluye toda una serie de interpretaciones y, en este sentido, ofrece muchas hipótesis sobre el significado y alcance de conceptos jurídicos, reglas, principios, etc., que pueden ser confirmados o falseados por medio de la investigación científica. Las cuestiones explícitas de interpretación no son un fenómeno marginal en el Derecho. Surgen cuando los textos no son claros. Pero también surgen cuando su interpretación literal nos aboca a resultados no razonables, inequitativos o absurdos. Confrontar ese resultado con el significado dado al texto, en algún sentido, "falsea" el significado implícito o prima facie del texto. Esto nos llevará a la formulación de una nueva hipótesis acerca del significado del texto, que será analizado por el investigador con una interpretación metodológica más concienzuda.

Una hipótesis a propósito del significado exacto de un concepto, norma o principio jurídico, no se refiere únicamente a indagar aquello que sus autores tenían en mente. El contexto normativo actual y el resultado socialmente deseable también co-determinan el significado. Por tanto, este significado evoluciona y puede cambiar en el curso de los años sin que medie cambio alguno en su texto. La unanimidad actual respecto al significado de un texto jurídico no obstaculiza que los académicos en el futuro formulen nuevas hipótesis sobre su significado, sea ligeramente diferente o radicalmente distinto.

La formulación de preguntas de investigación es libre. No hay reglas que la limiten. Evidentemente, las mismas deben tener sentido y adecuarse a las teorías paradigmáticas que sirven como marco para la investigación doctrinal en el sistema jurídico en cuestión (la teoría de las fuentes jurídicas, por ejemplo, o la teoría sobre los métodos de interpretación aceptables), a menos que el investigador pretenda cuestionar el marco teórico en sí.

\section{c) LA CONSTRUCCIÓN DE TEORÍAS EN LA DOCTRINA JURÍDICA}

Una "teoría científica" se define como "un sistema de afirmaciones, opiniones y conceptos coherentes, especialmente, no contradictorios, relativos a algún área de la realidad, que son formulados de tal manera que es posible deducir hipótesis contrastables de ellas" ${ }^{38}$

En doctrina jurídica, esto significaría:

"Una teoría en Derecho es un sistema de afirmaciones opiniones y conceptos coherentes, no contradictorios, relativos, a algún sistema jurídico o alguna parte de él, que son formulados de tal manera que es posible deducir de ellos hipótesis contrastables sobre la existencia (validez) e interpretación de conceptos, reglas o principios jurídicos"

Por ejemplo, desde la teoría del efecto directo del Derecho de la Unión Europea uno puede deducir hipótesis sobre la (in)validez y (re)interpretación de la legislación del sistema jurídico propio al interior de la Unión Europea.

Tales teorías están, a su vez, basadas en supuestos generalmente aceptados que crean el marco paradigmático de la doctrina jurídica. Esos supuestos compartidos incluyen: un entendimiento compartido sobre lo que el "Derecho" es y sobre su rol en la sociedad; una teoría de las fuentes jurídicas válidas y sobre su jerarquía; una metodología jurídica; una teoría de la argumentación; una teoría de la legitimación y una visión del mundo compar-

${ }^{38}$ DE GROOT, A. D., Methodologie, $3^{\text {a }}$. ed., The Hague, Mouton, 1966, p. 42. 
tida (valores y normas básicas compartidas). Dentro de la doctrina jurídica las anteriores son "meta-teorías", por lo que la definición de "teoría" anteriormente enunciada también es válida. Esos supuestos paradigmáticos están profundamente arraigados en la tradición, pero pueden evolucionar, y en algunos casos también cambiar. Ejemplo de ello son, dentro de la teoría de las fuentes jurídicas: la aceptación de la prioridad del Derecho de la Unión Europea sobre el Derecho interno de los Estados miembros, o la aceptación de "principios generales del Derecho implícitos” como fuente válida del Derecho. En la metodología jurídica, son ejemplos: la aceptación de un rol más activo del juez en la interpretación del Derecho. Respecto a la visión del mundo compartida: visiones cambiantes sobre el matrimonio, la familia, la homosexualidad, el aborto o la eutanasia. En cada periodo, los supuestos paradigmáticos de ese tiempo son constitutivos del marco dentro del cual teorías más concretas a propósito del Derecho son formuladas, comprobadas y discutidas entre la comunidad científica de investigadores jurídicos.

La fuerza de las teorías científicas radica en su capacidad para abarcar un dominio tan amplio como sea posible, con un marco sencillo de conceptos, reglas y principios y su capacidad para generar una gran cantidad de hipótesis comprobables. Para las disciplinas explicativas, la capacidad explicativa de una teoría es otro elemento para juzgar su fortaleza.

En una primera etapa, los conceptos se interpretan para ordenar la realidad. Esto implica la abstracción, la coherencia lógica y, en la medida de lo posible, la simplicidad. Uno puede, por ejemplo, asumir que en las sociedades primitivas los animales fueron clasificados inicialmente de conformidad a su tamaño o color y/o de acuerdo a su capacidad para volar, nadar o correr. Posteriormente, sin embargo, cuando el conocimiento teorético devino más accesible, la clasificación se hizo en base a otras divisiones: mamíferos/no-mamíferos o en base a sus similitudes o diferencias por lo que respecta a su estructura de ADN. De la misma manera, el desarrollo del Derecho y de la doctrina jurídica muestra un creciente nivel de abstracción. Como en el caso de las sociedades primitivas, las reglas se desarrollaron teniendo como base los casos concretos, que fueron inicialmente distintas reglas, por ejemplo, para el robo de un caballo, una vaca o una oveja. En un momento ulterior, el concepto más abstracto (el robo de) "ganado" se introdujo. En una siguiente etapa de abstracción, devino (el robo de) "bienes muebles". Conceptos tales como "vaca", "oveja" o "caballo" hacen referencia directa a objetos visibles en la realidad, mientras que un concepto como "ganado" es una abstracción basado en la utilidad de ciertos tipos de animales para el hombre, al igual que las "mascotas" o los "ciervos", que tienen otro tipo de utilidad para los seres humanos; mientras que, por el contrario, los "bichos" son carentes de utilidad para la humanidad. La amplia división general entre "bienes muebles" e "inmuebles" utiliza como criterio decisivo, a su vez, la movilidad y por tanto la negociabilidad de los objetos. Con la introducción de la distinción entre materias "corpóreas" e "incorpóreas", los "bienes" como objetos tangibles se amplían con "derechos" y otras abstracciones, que solamente pueden clasificarse como "bienes" dentro de un sistema jurídico desarrollado. Todas estas divisiones pueden parecer "naturales" para la mayoría de nosotros, pues hemos crecido con ellos y difícilmente podemos imaginarnos un sistema jurídico construido sobre la base de otras divisiones; como por 
ejemplo la división entre "ratas" y "no-ratas" que solía ser la división principal en el Derecho de la tribu Kapauku en Papúa, Nueva Guinea. ${ }^{39}$

En otras palabras, detrás de esos conceptos y divisiones, existe una visión del mundo subyacente así como la elección de valores, intereses y principios que explican las teorías y las divisiones que acompañan a esos conceptos. La realidad es descrita, comprendida, ordenada $-\mathrm{y}$, de hecho, en parte creada- mediante tales conceptos y redes de conceptos que traducen una cosmovisión subyacente. De hecho, los conceptos no son herramientas neutrales. Están guiados por una finalidad teórica. La formulación de conceptos y la construcción de teorías están estrechamente interrelacionados en cada disciplina. Tal y como señalara Hempel, se trata en realidad de dos caras de la misma actividad. ${ }^{40}$ Los conceptos traducen o trasladan divisiones de la realidad que se consideran más importantes o relevantes que otras. En biología, actualmente, la forma de procreación de los animales (mamíferos o no) se considera más importante que su biotipo (agua, aire o tierra). En el Derecho Romano la distinción "bienes muebles/inmuebles" fue el núcleo del Derecho Civil y no, por ejemplo, la distinción entre criaturas vivientes y no-vivientes. El criterio subyacente es la diferencia de su importancia económica entre las categorías: la propiedad, los "bienes inmuebles", constituían la parte principal de cada fortuna en Europa hasta el siglo XIX. Los bienes muebles tienen mucho menos valor en general. Hoy, sin embargo, la importancia económica de esta división legal ha sido en gran parte socavada por el surgimiento y desarrollo de las sociedades. Las empresas se han convertido en los dueños de importantes bienes inmuebles, mientras que las acciones de las mismas se consideran como bienes muebles. De esta forma, a través de la creación jurídica de empresas y firmas, los bienes inmuebles cambian a "muebles", con lo cual la importancia económica de esta división pierde su sentido. En el Código Napoleónico, en 1804, la distinción entre "bienes muebles" e "inmuebles" determinaba qué tipo de bienes, en las capitulaciones matrimoniales, se convertían en "comunes" (bienes muebles) para ambos cónyuges, y cuáles permanecían en propiedad exclusiva de cada uno de ellos (bienes inmuebles). ${ }^{41}$ Cuando, en 1976, el legislador belga cambió a fondo las capitulaciones matrimoniales, abandonó la regulación consistente en la división entre "bienes muebles" e "inmuebles", reemplazándola por la división entre los bienes que se poseían antes de la boda (que siguen siendo propios) y los bienes adquiridos durante el matrimonio (que se convierten en comunes). ${ }^{42}$ Esta división se adoptó teniendo como base otras visiones sobre el matrimonio, incluyendo la aceptación de sucesivos matrimonios y divorcios. Ya no se da por sentado que el matrimonio dura "hasta que la muerte nos separe". Tener relaciones sucesivas a lo largo de la vida se ha convertido en la regla más que en la excepción. Por tanto,

\footnotetext{
39 Como documentó: POSPISIL, L., Anthropology of Law. A Comparative Theory, New York, Joanna Cotler Books, 1971, pp. 274-302.

40 HEMPEL, C. G., Fundamentals of Concept Formation in Empirical Science, Chicago, The University of Chicago Press, 1952, p. 1 y ss.

${ }^{41}$ Esto representaba los bienes muebles e inmuebles que las esposas poseían en el día de su boda (artículos 1401 y 1404 del Código Napoleónico de 1804, lo cual se mantuvo inalterado durante casi dos siglos en muchos países -en Bélgica, por ejemplo, hasta 1976.)

42 Artículos 1399, 1405, 1406 y 1408 del Código Civil belga.
} 
hoy parece más razonable que los cónyuges compartan sólo aquello que adquirieron juntos, durante su matrimonio.

Así, los conceptos juegan un papel importante en la construcción de teorías, pero al mismo tiempo, son, en combinación con reglas y principios, resultado de esas teorías.

La construcción de teorías pretende combinar interpretaciones específicas de principios jurídicos, reglas y conceptos en un (nuevo) todo sistematizado. ${ }^{43}$ En doctrina jurídica, sistematización significa construcción de teorías. La re-sistematización del Derecho es una actividad continua de la investigación jurídica debido al crecimiento de la legislación y de la jurisprudencia. Por ejemplo, nueva legislación en el área de protección al consumidor, precisa de alguna re-sistematización del Derecho de contratos. Nuevas interpretaciones judiciales pueden requerir una optimización, o incluso un cambio radical, de teorías previas. Nuevas reglas europeas, que afectan directamente al Derecho nacional, pueden requerir de la re-sistematización de las áreas jurídicas afectadas en los sistemas jurídicos nacionales de los estados miembros.

\section{CONCLUSIÓN}

En este breve itinerario sobre la naturaleza y metodología de la investigación jurídica, la doctrina jurídica se nos presenta como una disciplina científica por derecho propio con una metodología que, en sus características primordiales, es bastante comparable con la metodología utilizada en otras disciplinas.

Sin embargo, de ese recorrido también se desprende que no existe un acuerdo entre los teóricos jurídicos sobre la naturaleza de la doctrina jurídica como disciplina; tal desacuerdo se da independientemente, incluso, de las diferencias entre las tradiciones nacionales de investigación jurídica. Partiendo de la forma en que la doctrina jurídica se ha practicado en el curso de la historia en la mayoría de los países, desde la época romana, podemos considerarla como una disciplina fundamentalmente hermenéutica; una disciplina que cuenta también con elementos empíricos, argumentativos, lógicos y normativos. La descripción en el Derecho está estrechamente vinculada a su interpretación y, cuando describe el Derecho, el jurista está formulando hipótesis sobre su existencia, validez y significado. El nivel de sistematización y conceptualización es el nivel de la construcción de teorías en la doctrina jurídica.

Algunos temas que no han podido discutirse en el presente trabajo pero que quedan abiertos para futuras investigaciones y debate, propongo, son los siguientes:

- La estrecha relación entre la práctica y la doctrina jurídica, incluida la producción científica llevada a cabo por abogados litigantes (de tiempo completo) y la de algunos investigadores activos en la abogacía, hacen que sea difícil trazar la línea entre las dos, así como entre las publicaciones que son "científicas" y las de mera "divulgación", es decir, transmisión de información sobre alguna área del Derecho.

- Obviamente, teorías divergentes del significado, co-determinan la existencia de teorías divergentes sobre la naturaleza de la investigación jurídica científica y su metodología

\footnotetext{
${ }^{43}$ Véase, como ejemplo de lo estrechamente relacionados que están la interpretación y sistematización en el Derecho: CHEVALLIER, J., "Conclusion générale. Les interprètes du droit" en: Y. Poirmeur, et. al, La doctrine juridique, Paris, Presses Universitaires de France, 1993, p. 276.
} 
apropiada. Parece dudoso que pueda llegarse a un consenso sobre esas teorías del significado puede ser alcanzado dentro de la comunidad jurídica científica internacional o, incluso, dentro de una comunidad de investigadores jurídicos pertenecientes a un solo sistema jurídico. Por lo tanto, será aún más difícil llegar a un de consenso de ese tipo sobre la naturaleza y metodología de la doctrina jurídica.

- El problema de la "objetividad" en el ámbito de la investigación jurídica, ya que no existe una "realidad objetiva" más allá o independiente de las construcciones de la doctrina jurídica. Además, los investigadores jurídicos a menudo toman posiciones normativas o valorativas; colocan alguna preferencia entre valores e intereses, siendo ello subjetivo par excellence. Más allá de las ciencias exactas, la única forma de "objetividad" que uno puede alcanzar es el consenso intersubjetivo entre los teóricos del Derecho.

- No toda investigación trata de probar hipótesis o de construir teorías. Existen, por ejemplo en una etapa preparatoria, también "preguntas de diagnóstico" o una "investigación puramente descriptiva” (v. gr. la recolección de datos con el fin de formular una hipótesis en una etapa posterior, y no su simple divulgación).

- No porque la descripción/interpretación del Derecho y la sistematización/construcción de teorías hayan sido siempre el núcleo básico de la doctrina jurídica, uno no debe considerar ampliarlo, en una dirección más multidisciplinar, colocando al Derecho en un contexto de un modo más sistemático y usar metodologías adecuadas de otras disciplinas. La pregunta aquí, por supuesto, es la siguiente: ¿hasta dónde deben llegar los investigadores jurídicos en este esfuerzo y dónde alcanzan estos su punto de incompetencia? La conformidad con el enfoque de las ciencias empíricas no es una buena razón para hacerlo, pero con la finalidad simplemente de comprender el Derecho y elaborar teorías y conceptos jurídicos, uno requiere este enfoque contextual del Derecho.

[traducción de Francisco M. Mora Sifuentes]

\section{FUENTES}

AARNIO, A., Philosophical Perspectives in Jurisprudence, Acta Philosophica Fennica, Helsinsky, Academic Bookstore, 1983.

ALBERT, H., "Kennis en Recht” en: F. D. Heyt (ed.), Rationaliteit in wetenschap en samenleving, Alphen ann de Rijn, Samsom, 1976.

, Traktat über rationale Praxis, Tübingen, J. C. B Mohr, 1978.

ALEXY, R., Theorie der Juristischen Argumentation, Suhrkamp Verlag, Frankfurt am Main, 1983 [trad. cast. de M. Atienza e I. Espejo, Teoría de la argumentación jurídica, Madrid, CEPC, $2^{\mathrm{a}}$ ed., 2007].

ANDRÉ, A., "Was heisst rechtswissenschafliche Forschung?", Juristenzeitung, 1970, pp. 396-401. 
BROCKMÖLLER, A., Die Entstehung der Rechtstheorie im 19. Jahrhundert in Deutschland, Baden-Baden, Nomos Verlag, 1997.

CHEVAlLiER, J., "Conclusion générale. Les interprètes du droit” en: Y. Poirmeur, et. al, La doctrine juridique, Paris, Presses Universitaires de France, 1993.

DE GEEST, G., "Hoe maken we van de rechtswetenschap een volwaardige wetenschap?, Nederlands Juristenblad, 2004, pp. 58-66.

DE GROOT, A. D., Methodologie, 3ª ed., The Hague, Mouton, 1966.

DWORKIN, R., Law's Empire, London, Fontana Press, 1986.

FINN, DAVID D., "Magnitude and Importance of Legal Science” (1859), en: Presser, S. B., y Zainaldin, J. S. (eds.), Law and Jurisprudence in American History, Minnesota, West Publishing Co., 1995.

GREENLEAF, S., A Discourse Pronounced at the Inauguration of the Authors as Royall Professor of Law in Harvard University, Cambridge, Massachusetts, James Munroe, 1834.

GOFF OF CHIEVELY, Lord R. L. A., "Judge, Jurist and Legislature”, Denning Law Journal, núm. 2, 1987, pp. 79-95.

HOLMES, O. W., "The Path of the Law”, Harvard Law Review, núm. 10, 1897, pp. 457-478 [trad. cast. y est. preliminar de J. I. Solar Cayón, La senda del Derecho, Madrid, Marcial Pons, 2012].

HUGO, G., Lehrbuch eines civilistischen Cursus, Philosophische Encyclopedie für Juristen, Berlin, 1799, vol. 5 .

KELSEN, H., General Theory of Norms, Oxford, Clarendon Press, 1991. [trad. cast. de H. C. Delory, rev. técnica de J. F. Arriola, Teoría general de las normas, México, Trillas, 1994].

KRANENBURG, R., De Grondslagen der Rechtswetenschap. Jurdische kennisleer en methodologie, $5^{\text {a }}$ ed., Harleem, HD Tjeenk Willink, 1955.

LOTH, M. A., "Regel-geleid gedrag; over het object van empirische rechtswetenschap", Rechtsfilosofie \& Rechtstheorie. Netherlands Journal for Legal Philosophy and Jurisprudence, núm. 3, 1983, pp. 213-228.

LUNDSTEDT, A. V. Die Unwissenschaftlichkeit der Rechtswissenschaft, Berlin, Grunewald, W Rothschild, vol I., 1932.

LYALL, A., "Early German Legal Antropology: Albert Hermann Post and His Questionnaire", Journal of African Law, núm. 52, 2008, pp. 114-124 (con el cuestionario como apéndice en pp. 124-138).

MOÓR, J. “Das Logische im Recht”, Revue Internationale de la Théorie du Droit/Internationale Zeitschrift für Theorie des Rechts, 1927-1928, pp. 157-203.

MULDER, T., Ik beschulding de rechtsgeleerde faculteit van onwetenschappelijkheid, Leiden, 1937. 
NAGEL, E., "The Nature and Aim of Sciente", en: S. Morgenbesser (ed.), Philosophy of Science Today, New York, Basic Books, 1967.

PECZENIK, A., "Scientia Juris. Legal Doctrine as Knowledge of Law and as a Source of Law”, en: E. Pattaro (ed.), A Treatise of Legal Philosophy and General Jurisprudence, Dordrecht, Springer, 2005, vol. 4

PERELMAN, CH., Logique juridique. Nouvelle rhétorique, Paris, Dalloz, 1976 [trad. cast. de L. Diez-Picazo, La lógica jurídica y la nueva retórica, Madrid, Civitas, 1979].

POSPISIL, L., Anthropology of Law. A Comparative Theory, New York, Joanna Cotler Books, 1971.

POST, A. H., Einleitung in eine Naturwissenschaft des Rechts, Oldemburg, Verlag der Schulzchen Buchhandlug, 1872.

ROSS, A., On Law and Justice, London, Stevens \& Sons, 1958 [trad. cast. de G. R. Carrió, Sobre el derecho y la justicia, Editorial Universitaria de Buenos Aires, Bs. As., 1963].

SAMUEL, G., "Is Law Really a Social Science? A View from Comparative Law", Cambridge Law Journal, núm. 67, 2008, pp. 288-312.

SCHWEBER, H., "Law and the Natural Sciences in Nineteenth-Century American Universities”, en: S. S. Silbey (ed.), Law and Science, The International Library of Essays in Law and Society, Aldershot, Ashgate, 2008, pp. 3-23.

SMITH, C., et al., "Criteria voor goed rechtswetenschappelijk onderzoek", Nederlands Juristenblad, 2008, pp. 685-690.

SMITS, J., "Redefining Normative Legal Science: Towards an Argumentantive Discipline” en: F. Grünfeld et al y F. Commands (eds)., Methods of Human Rights Research, Antwerp, Intersentia, 2009.

STEINMETZ, S. R., Ethnologische Studien zur ersten Etwicklung der Strae, $2^{\mathrm{a}}$ ed., 2 vols., Groningen, P. Noordhoff, 1928, (la primera edición se publicó en Leiden en 1894).

, Rechtsverhältnisse von eingeborenen Völkern in Afrika und Ozeanien. Beantwortungen des Fragebogens der Internationalen Vereinigung für vergleichende Rechtswissenchaft und Volkswirtschaftslehre zu Berlin, Berlin, Julius Springer, 1903.

STRUCK, G., Topische Jurisprudenz, Frankfurt, Athenäun Verlag, 1971.

TEBALDESCHI, I., Rechtswissenschaft als Modellwissenchaft, Viena, Springer Verlag, 1979.

THIERAU, J.-L., "La doctrine civiliste avant le Code civil” en: Y. Poirmeur , et al., La doctrine juridique, Paris, Presses Universitaires de France, 1993.

TWINING, W., Blackstone's Tower: The English Law School, London, Sweet \& Maxwell, 1994.

VAN DEN BERGH, G.C.C.J., Geleerd recht. Een geschiedenis van de Europese rechtswetenschap in vogelvlucht, $2^{\mathrm{a}}$. ed., Deventer, Kluwer, 1985

VAN HOECKE, M., Law as Communication, Oxford, Hart Publishing, 2002. 
VAN HOUTEN, S., Das Causalitätsgesetz in der Socialwissenschaft, Haarlem, HD Tjeenk Willink and Leipzig, FA Brockhaus, 1888.

VAN QUICKENBORNE, M., "Rechsstudie als wetenschap" en: Actori incubit probatio, Antwerp, Kluwer, 1975.

VICK, D. W., "Interdisciplinarity and the Discipline of Law", Journal of Law and Society, núm. 31, 2004, pp. 163-193.

VIEHWEG, TH., Topik und Jurisprudenz: ein Beitragzur Rechtswissenschaftli-chen Grundlagenforschung, 5a. ed. München, Beck Vergag 1974 [trad. cast. de L. Diez-Picazo Ponce de León y prólogo de E. García de Enterría, Tópica y jurisprudencia, Madrid, Civitas, reimp. 2004]

VON KIRCHMANN, J. Die Werthlosigkeit der Jurisprudenz als Wisseschaft, Berlin, Julius Springer, 1848 [trad. cast. y estudio preliminar de A. Truyol y Sierra, La jurisprudencia no es ciencia, $3^{\text {a }}$. ed., Madrid, CEC, 1983].

VON WRIGHT, G. H., Explanation and Understanding, Ithaca, Cornell University Press, 1971. [trad. cast. de L. Vega Reñón, Explicación y comprensión, Madrid, Alianza Editorial, $1^{\text {a }}$. reimp. 1987]

WENDT, J. A. I., De methode der rechtswetenschap vanuit kritisch-rationeel perspectief, Zutphen, París, 2008.

WILKEN, G. A., De vrucht van de beoefening der ethnologie voor de vergelikende rechtswetenschap, (Inaugural Lecture, Rijksuniversiteit Leiden), Leiden, EJ Brill, 1885. 